\title{
Content-Based 3D Mosaics for Dynamic Urban Scenes
}

\author{
Zhigang Zhu*a, Hao Tang ${ }^{a}$, George Wolberg ${ }^{a}$ and Jeffery R. Layne ${ }^{\mathrm{b}}$ \\ ${ }^{a}$ Department of Computer Science, City College of New York, New York, NY 10031, USA \\ ${ }^{\mathrm{b}}$ Air Force Research Laboratory, WPAFB, Ohio 45433-7318, USA \\ *zhu@cs.ccny.cuny.edu
}

\begin{abstract}
We propose a content-based 3D mosaic (CB3M) representation for long video sequences of 3D and dynamic scenes captured by a camera on a mobile platform. The motion of the camera has a dominant direction of motion (as on an airplane or ground vehicle), but 6 DOF motion is allowed. In the first step, a set of parallel-perspective (pushbroom) mosaics with varying viewing directions is generated to capture both the $3 \mathrm{D}$ and dynamic aspects of the scene under the camera coverage. In the second step, a segmentation-based stereo matching algorithm is applied to extract parametric representations of the color, structure and motion of the dynamic and/or 3D objects in urban scenes where a lot of planar surfaces exist. Multiple pairs of stereo mosaics are used for facilitating reliable stereo matching, occlusion handling, accurate $3 \mathrm{D}$ reconstruction and robust moving target detection. We use the fact that all the static objects obey the epipolar geometry of pushbroom stereo, whereas an independent moving object either violates the epipolar geometry if the motion is not in the direction of sensor motion or exhibits unusual 3D structures. The CB3M is a highly compressed visual representation for a very long video sequence of a dynamic 3D scene. More importantly, the CB3M representation has object contents of both 3D and motion. Experimental results are given for the CB3M construction for both simulated and real video sequences to show the accuracy and effectiveness of the representation.
\end{abstract}

Keywords: Multi-image registration, content-based video coding, image-based modeling, video surveillance

\section{INTRODUCTION}

In this paper we address the problems of visual representation for large amount of video stream data, of threedimensional (3D) urban scenes in particular, captured by a camera mounted on an airborne or a ground mobile platform. Applications include airborne or ground video surveillance for moving target extraction, automated 3D urban scene construction, airborne traffic monitoring, and image-based modeling and rendering. For these applications, hours of video streams may be generated every time the mobile platform performs a data collection task. The data amount is in the order of $100 \mathrm{~GB}$ per hour for standard $640 * 480$ raw color images. The huge amount of video data not only poses difficulties in data recording and archiving but also is prohibitive for users to retrieve and to review. In the past, video mosaic approaches ${ }^{1,2,3}$ have been proposed for video representation and compression, but most of the work is for panning (rotating) cameras instead of moving (translating) cameras that are mostly used in the cases of airborne or ground mobile surveillance and scene modeling. In those applications, obvious motion parallax is the main characterization of the video sequences due to the self-motion of the sensors. Some work has been done in 3D reconstruction of panoramic mosaics ${ }^{5,6}$, but usually the results are 3D depth maps of static scenes instead of high-level $3 \mathrm{D}$ representations for both static and dynamic target extraction and indexing. Layered representations ${ }^{7,8,9}$ have been studied for motion sequence representations; however, the methods are usually computationally expensive, and the outputs are typically motion segmentation represented by affine planes instead of true 3D information. Efficient, highlevel, content-based, and very low bit-rate representations of 3D scenes and moving targets are still in great demand.

We propose a content-based 3D mosaic representation (CB3M) for long video sequences of 3D and dynamic scenes captured by a camera mounted on a mobile platform. The motion of the camera has a dominant direction of motion (as on an airplane or ground vehicle), but 6 DOF motion is allowed. In the first step, a set of generalized parallel-perspective (pushbroom) mosaics with varying viewing directions is generated to capture both the $3 \mathrm{D}$ and dynamic aspects of the scene under the camera coverage. Bundle adjustment techniques ${ }^{14}$ can be used for camera pose estimation, sometimes integrated with the geo-referenced data from GPS and INS when available. A ray interpolation approach ${ }^{10}$ is used to generate multiple seamless parallel-perspective mosaics under the obvious motion parallax of a translating camera. The 
set of the multi-view dynamic pushbroom mosaics, with a pair of stereo mosaics as the minimum sub-set, is a compact visual representation for a long video sequence of a $3 D$ scene with independent moving targets.

However, the 2D mosaic representation is still an image-based one without object content representation. Therefore, in the second step, a segmentation-based stereo matching algorithm ${ }^{11}$ is applied to extract parametric representation of the color, structure and motion of the dynamic and/or 3D objects in urban scenes, where a lot of planar surfaces exist. In the algorithm, we use the fact that all the static objects obey the epipolar geometry, i.e. along the epipolar lines of pushbroom stereo. An independent moving object (moving on a road surface), on the other hand, either violates the epipolar geometry if the motion is not in the direction of sensor motion or exhibits unusual 3D structure, e.g., obviously hanging above the road or hiding below the road. Further in this paper, multiple pairs of stereo mosaics are used for facilitating reliable stereo matching, occlusion handling, accurate 3D reconstruction and robust moving target detection.

Based on the above two steps, a content-based 3D mosaic (CB3M) representation is created for the long video sequence. This is a highly compressed visual representation for the video sequence of a dynamic 3D scene. For example, a real image sequence of a campus scene has 1000 frames of $640 * 480$ color images. With its CB3M representation, a compression ratio of more than 10,000 is achieved. More importantly, the CB3M representation has object contents. We will also show the accuracy of $3 \mathrm{D}$ reconstruction and moving target detection by using a simulated video sequence while ground truth data is available.

The rest of the paper is organized as the follows. In Section 2, the mathematical framework of the dynamic pushbroom stereo is given, and then its properties for moving target extraction are discussed. In Section 3, multi-view pushbroom mosaics are proposed for image-based rendering and for extracting 3D structure and moving targets. In Section 4, our multi-view stereo matching algorithm for 3D static and moving target extraction will be provided. Then in Section 5, the content-based 3D mosaic representation is described. Experimental results of CB3M representation construction will be given in Section 6 with both simulated and real video data. Section 7 gives concluding remarks.

\section{DYNAMIC PUSHBROOM STEREO MOSAICS}

For show the concept, let us first assume the motion of a camera is an ideal 1D translation, the optical axis is perpendicular to the motion, and the frames are dense enough. Then, we can generate two spatio-temporal images by extracting two columns of pixels (perpendicular to the motion) at the leading and trailing edges of each frame in motion (Fig. 1). The mosaic images thus generated are parallel-perspective, which have perspective projection in the direction perpendicular to the motion and parallel projection in the motion direction. In addition, these mosaics are obtained from two different oblique viewing angles of a single camera's field of view, so that a stereo pair of left and right mosaics captures the inherent 3D information. The geometry in this ideal case (i.e. 1D translation with constant speed) is the same as the linear pushbroom camera model ${ }^{15}$. Therefore we also call this image representation pushbroom stereo mosaics.

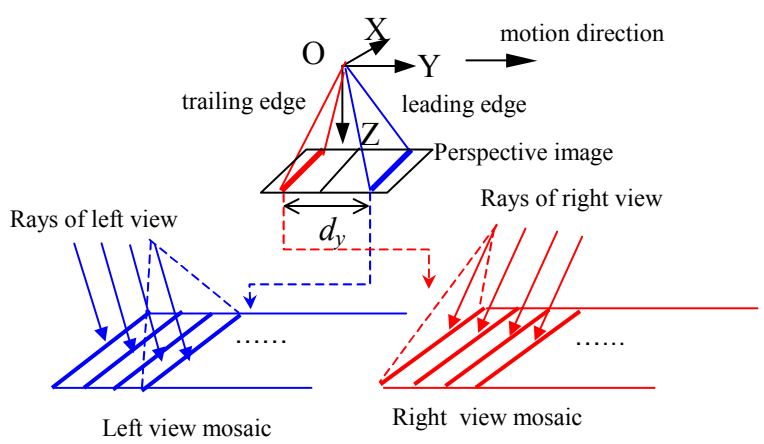

Fig. 1. Principle of the pushbroom stereo mosaics

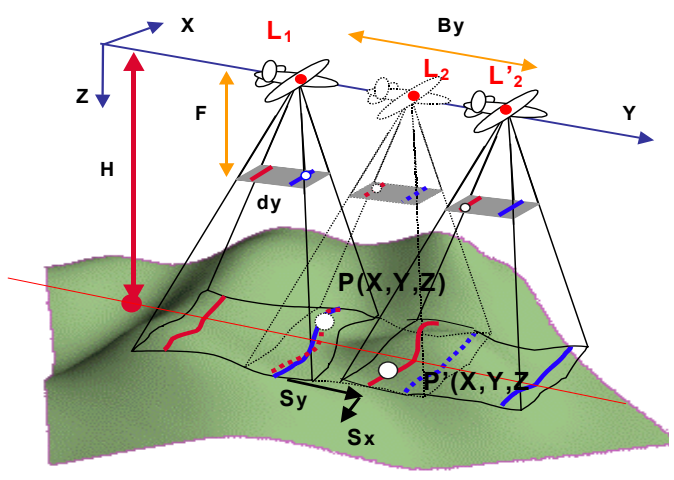

Fig. 2. Dynamic pushbroom stereo mosaics 
In real applications, there are two challenging issues. The first problem is that the camera usually cannot be controlled with ideal 1D translation and camera poses are unknown; therefore, camera orientation estimation (i.e., dynamic calibration) is needed. In our previous study on an aerial video application, we used external orientation instruments, i.e., GPS, INS and a laser profiler, to ease the problem of camera orientation estimation ${ }^{10,16}$. More general approaches using bundle adjustment techniques ${ }^{14}$ are under investigation for efficiently estimating camera poses of long image sequences. In this paper, we assume that the extrinsic and intrinsic camera parameters are known at each camera location. The second problem is to generate dense parallel mosaics with a sparse, uneven, video sequence, under a more general motion, and for a complicated 3D scene. To solve this problem, we have proposed a parallel ray interpolation for stereo mosaics (PRISM) approach ${ }^{10}$ for generating a generalized stereo mosaic representation under constrained 6 DOF motion.

In principle, the PRISM approach needs to match all the points between the two overlapping slices of the successive frames to generate a complete parallel-perspective mosaic. In an effort to reduce the computational complexity, a fast PRISM algorithm ${ }^{10}$ has been designed, based on the proposed PRISM method. It only requires matches between a set of point pairs in two successive images, and the rest of the points are generated by warping a set of triangulated regions defined by the control points in each of the two images. The proposed fast PRISM algorithm can be easily extended to use more feature points (thus smaller triangles) in the overlapping slices so that each triangle really covers a planar patch or a patch that is visually indistinguishable from a planar patch, or to perform pixel-wise dense matches to achieve true parallel-perspective (pushbroom) geometry.

Dynamic pushbroom stereo mosaics ${ }^{11}$ are generated in the same way as with the static pushbroom stereo mosaics described above. Fig. 2 illustrates the geometry. A 3D point $\mathrm{P}(X, Y, Z)$ on a target is first seen through the leading edge of an image frame when the camera is at location $\mathrm{L}_{1}$. If the point $\mathrm{P}$ is static, we can expect to see it through the trailing edge of an image frame when the camera is at location $\mathrm{L}_{2}$. The distance between leading and trailing edges is $d_{y}$ (pixels), which denotes the constant "disparity" between this pair of images. However, if the point P moves during that time, the camera needs to be at a different location L' 2 to see this moving point through its trailing edge. For simplifying equations, we assume that the motion of the moving point between two observations $\left(\mathrm{L}_{1}\right.$ and $\left.\mathrm{L}_{2}{ }_{2}\right)$ is a $2 \mathrm{D}$ motion $\left(S_{x}, S_{y}\right)$, which indicates that the depth of the point does not change over that period of time. Therefore, the depth of the moving point can be calculated as

$$
Z=F \frac{B_{y}-S_{y}}{d_{y}}
$$

where $F$ is the focal length of the camera and $B_{y}$ is the distance of the two camera locations (in the $y$ direction). Mapping this relation into stereo mosaics following the notation in $^{10}$, we have

$$
Z=H\left(\frac{d_{y}+\Delta y-s_{y}}{d_{y}}\right)
$$

and

$$
\left(S_{x}, S_{y}\right)=\left(Z \frac{s_{x}}{F}, H \frac{s_{y}}{F}\right)=\left(Z \frac{\Delta x}{F}, H \frac{s_{y}}{F}\right)
$$

where $H$ is the depth of plane on which we want to align our stereo mosaics, $(\Delta x, \Delta y)$ is the visual motion of the moving 3D point $\mathrm{P}$, which can be measured in the stereo mosaics. The vector $\left(s_{x}, s_{y}\right)$ is the target motion represented in stereo mosaics. Obviously, we have $s_{x}=\Delta x$. The above analysis only shows the geometry of a moving camera with 1D translational motion. In fact, a pair of generalized stereo mosaics can be generated when the camera undertakes constrained 6 DOF motion. Details of the representation and algorithms can be found in our previous work ${ }^{10}$.

We have the following interesting observations about the dynamic pushbroom stereo geometry for 3D and moving target extraction.

(1) Stereo fixation. For a static point (i.e. $\left.S_{x}=S_{y}=0\right)$, the visual motion of the point with a depth $H$ is $(0,0)$, indicating that the stereo mosaics thus generated fixate on the plane of depth $H$. This fixation facilitates the stereo matching and the detection of moving targets on that plane. 
(2) Motion accumulation. For a moving point $\left(S_{x} \neq 0\right.$ and/or $\left.S_{y} \neq 0\right)$, the motion between two observations accumulates over a period of time due to the large distance between the leading and trailing edges in creating the stereo mosaics. This will increase the discrimination capability for slowly moving objects viewed from a relatively fast moving aerial camera. (3) Epipolar constraints. In the ideal case of 1D translation of the camera (with which we present our dynamic pushbroom stereo geometry in this paper), the correspondences of static points are along horizontal epipolar lines, i.e., $\Delta x=0$. (As a generalization, an epipolar curve geometry under 3D camera motion is given in our previous paper ${ }^{10}$.) Therefore, for a moving target $\mathrm{P}$, the visual motion with nonzero $\Delta x$ (i.e., the visual motion in the $x$ direction) will identify itself from the static background in the general case, which implies that the motion of the target in the $\mathrm{x}$ direction is not zero (i.e., $S_{x} \neq 0$ ). In other words, the correspondence pair of such a point will violate the epipolar line constraint for static points (i.e. $\Delta x=0$ ).

(4) $3 D$ constraints. Even if the motion of the target happens to be in the direction of the camera's motion (i.e., the $y$ direction), we can still discriminate the moving target by examining 3D anomalies. Typically, a moving target (a vehicle or a human) moves on a flat ground surface (i.e., road) over the time period during which it is observed through the leading and trailing edges of video images with a limited field of view. We can usually assume that the moving target share the same depth as its surroundings, given that the distance of the camera from the ground is much larger than the height of the target. (The method to deal with $3 \mathrm{D}$ structure of a moving target is discussed in our previous work ${ }^{11}$.) $\mathrm{A}$ moving target in the direction of camera movement, when treated as a static target, will show 3D anomaly - either hanging up above the road (when it moves to the opposite direction, i.e., $S_{y}<0$ ), or hiding below the road (when it moves in the same direction, i.e., $\left.S_{y}>0\right)$.

After a moving target has been identified, the motion parameters of the moving target can be estimated. We first estimate the depth of its surroundings and apply this depth $Z$ to the target, then calculate the object motion $s_{y}$ using Eq. (2) and $\left(S_{x}\right.$, $\left.S_{y}\right)$, using Eq. (3), given the visual motion $(\Delta x, \Delta y)$ measured in the stereo mosaics.

\section{MULTI-VIEW PUSHBROOM MOSAICS}

A pair of stereo mosaics (generated from the leading and trailing edges) is a very efficient representation for both 3D structures and target movements. However, there are two remaining issues. First, stereo matching will be difficult due to the largely separated parallel views of the stereo pair. Second, for some unusual target movements, e.g. moving too fast, changing speed or direction, we may either have two rather different images in the two mosaics (if changing speed), or see the object only once (if changing direction), or never see the object (if it maintains the same speed as the camera and thus never shows up in the second edge window).

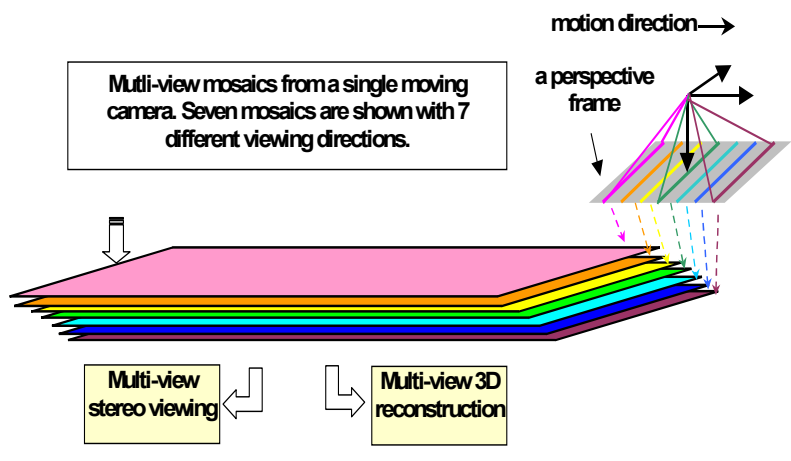

Fig. 3. Multi-view pushbroom mosaics

Therefore we propose to generate multi-view mosaics (more than 2), each of them with a set of parallel rays whose viewing direction is between the leading and the trailing edges (Fig. 3). The multiple mosaic representation is still efficient. Moreover, there are three benefits of using them. First, it eases the stereo correspondence problem in the same way as the multi-baseline stereo ${ }^{17}$, particularly for more accurate $3 \mathrm{D}$ estimation and occlusion handling. Second, multiple pushbroom mosaics can be used for image-based rendering ${ }^{21}$ with stereo viewing in which the translation across the area is simply a shift of a pair of mosaics, and the change of viewing directions is simply a switch between two consecutive 
pairs of mosaics. Third, multiple mosaics can also facilitate 3D estimation of moving targets ${ }^{11}$, and increase the possibility to detect moving targets with unusual movements and also to distinguish the movements of the specified targets (e.g., ground vehicles) from those of trees or flags in wind. In the next section, we will discuss a new method to extract both of 3D structures and moving targets from multiple dynamic pushbroom mosaics.

\section{3D AND MOTION CONTENT EXTRACTION}

Based on the observations in Section 2, our previous work of segmentation-based stereo matching ${ }^{11}$ integrates the estimation of 3D structure of an urban scene and the extraction of independent moving objects from a pair of dynamic pushbroom stereo mosaics. In this paper, we use the advantageous properties of multi-view mosaics, and propose a multi-view approach to perform both stereo matching and motion detection. In a set of pushbroom mosaics generated from a video sequence, the leftmost mosaic is used as the reference mosaic, therefore color segmentation is performed on this mosaic, and the so called natural matching primitives are extracted. Multiple natural matching primitives are defined with each homogeneous color image patch approximately corresponding to a planar patch in $3 \mathrm{D}$. The representations are effective for both static and moving targets in man-made urban scenes with objects of largely textureless regions and sharp depth boundaries. Then matches of those patches with natural matching primitives are searched in the rest of the mosaics, one by one. After matching each stereo pair, a plane is fitted for each patch, and a set of planar parameters for the planar patch is estimated. Then multi-view matches are performed, and therefore multiple sets of parametric estimates for this planar patch are obtained. The best set is selected as the final result for this patch by comparing match evaluation scores.

The multi-view dynamic stereo mosaic approach has the following five stages: (1) color segmentation and interest point extraction; (2) three-step stereo matching; (3) plane estimation from multiple views; (4) plane merging and (5) moving object extraction. We will describe the approach in detail in the following subsections.

\subsection{Patch and interest point extraction}

First, the reference mosaic of the stereo mosaic pair, i.e. the leftmost mosaic, is segmented, using the mean-shift-based approach proposed by Comanicu \& Meer ${ }^{12}$. The segmented image consists of image regions (patches) with homogeneous color, and each of them is assumed to be a planar region in 3D space. For each patch, the boundary is defined as a closed curve. Then we use a line fitting approach to extract feature points for stereo matching. The boundary of each patch is first fitted with connected straight-line segments using an iterative curve splitting method. The connecting points between line segments are defined as interest points, around which the natural matching primitives are going to be defined. Now we are ready to perform the three-step stereo match.

\subsection{Three-step stereo match}

A three-step matching algorithm is designed and applied to the first stereo mosaic pair. Let the leftmost (reference) mosaic and the second (target) mosaic be denoted as $I_{1}$ and $I_{2}$, respectively. The matching process consists of the following three steps.

Step 1: Global match. Since there are many frontal surfaces in a typical urban scene, a 2D translational vector is first obtained for each patch. For a frontal or near-frontal surface, all the pixels inside the patch (region) have similar visual displacements. Therefore, for each region in the mosaic $\mathrm{I}_{1}$, the sum of absolute difference (SAD) is carried out for all pixels in this region between the two mosaics $I_{1}$ and $I_{2}$ with a predefined search range. Thus the initial visual displacement of the region between $\mathrm{I}_{1}$ and $\mathrm{I}_{2}$ is obtained as the one minimizing the SAD.

Step 2: Local match. Since not all regions are frontal planes in 3D space, the pixels in each region do not have a fixed visual displacement. Thus, for each interest point extracted from the line fitting approach, the best match is searched within a neighborhood area of the initial visual displacement. Instead of using the conventional window-based match, we define the so-called natural matching primitives to conduct a sub-pixel stereo match. We define a region mask $M$ of size $m \times m$ centered at that interest point such that

$$
M(i, j)=\left\{\begin{array}{l}
1, \text { if }(\mathrm{x}+\mathrm{i}, \mathrm{y}+\mathrm{j}) \in \mathbf{R} \\
0, \text { otherwise }
\end{array}\right.
$$


The size $\mathrm{m}$ of the natural window is adaptively changed depending on the size of the region $\mathrm{R}$. In order that a few more pixels (1 2) around the region boundary (but not belonging to the region) are also included so that we have sufficient image features to match, a dilation operation is applied to the mask $M$ to generate a region mask covering pixels across the depth boundary. The weighted cross-correlation, based on the natural window centered at the point $(x, y)$ in the reference mosaic, is defined as

$$
C(\Delta x, \Delta y)=\frac{\sum_{i, j} M(i, j) I_{1}(x+i, y+j) I_{2}(x+i+\Delta x, y+j+\Delta y)}{\sum_{i, j} M(i, j)}
$$

Note that we still carry out correlation between two color images but only on those interest points on each region boundary, and only with those pixels within the region and on the boundaries. A sub-pixel search is performed in order to improve the accuracy of 3D reconstruction; in our experiments, the steps for shifting the natural window (i.e., the intervals between two successive $\Delta y$ choices) are 0.1 pixels. A match is marked as reliable if it passes the crosscheck proposed in Scharstein \& Szeliski (2002) ${ }^{18}$.

Step 3: Surface fitting. Assuming that each homogeneous color region is planar in 3D, a 3D plane $a X+b Y+c Z=d$, which is represented in the camera coordinate system as shown in Fig. 2, is fitted to each region after obtaining the 3D coordinates of the interest points of the region using the pushbroom stereo geometry (Eq. 2). We use a RANSAC method $^{19}$ to perform plane fitting. At each iteration, three interest points that are not co-linear are randomly selected to fit a plane, then the result is evaluated by warping all the interest points with reliable matches to the target mosaic. For each reliable matched interest point, if its warped location in the target mosaic is close to that determined by the local match, i.e. their distance is smaller than 1 pixel, then it is called a reliable and accurate match point (RAP). If the number of the RAPs is large enough (e.g., the ratio between the number of RAPs and that of all the reliable matched interest points is larger than or equal to 0.65 ), then the fitted plane is considered a good estimate.

\subsection{Plane parameters from multiple mosaics}

After the above three steps are applied to the first pair of stereo mosaics, initial estimations of the 3D structure of all the patches (regions) in the reference mosaic are obtained. Further matches between the reference mosaic and each of the rest of the mosaics are then conducted. However the global match (Step 1 in the section 4.2) is not applied; instead, the initial visual displacement of each interest point on a patch is predicted from the result of this point estimated from the first stereo pair. From Eq. (2), we know the visual displacement $\Delta y$ is proportional to the selected "disparity" $\left(d_{y}\right)$ for a pair of stereo mosaics for any static point (i.e., $s_{y}=0$ ). Therefore the visual displacement of the interest point in consideration can be predicted except that the point is on a moving object that (1) does not move along the epipolar line of the pushbroom stereo; or (2) moves along the epipolar line, but with a varying speed. Those points will be reconsidered in the region merging and moving target detection stages. For refining the initial estimates of visual displacements based on the predictions from the results of the first pair of stereo mosaics, Steps 2 and 3 in Section 4.2 are performed to obtain new plane parameters for each pair of stereo mosaics starting from the second pair.

Suppose there are $N$ pairs of stereo mosaics, constructed from $N+1$ pushbroom mosaics. Then $N$ sets of plane parameters $\left(a_{k}, b_{k}, c_{k}, d_{k}\right), k=1, \ldots, N$, are obtained for each region (patch) in the reference mosaic. In order to obtain the most accurate plane parameters for each planar patch, the following steps are performed. First, for each pair of stereo mosaics, the patches in the reference mosaic are warped to the target mosaic in order to compute a color sum of absolute differences (SAD) for each region, between warped and original target images. Then, among all the estimates for each patch, the set of plane parameters with the least SAD value is selected as the best plane estimate. Note that using the knowledge of plane structure (i.e., 3D orientation), the best angle to view the region can be estimated, where the viewing direction of the selected mosaic (among all the possible viewing directions) is as close as to the plane norm direction. Incorporating this information, the SAD calculations are only carried out for those patches between the reference and target mosaics if the plane norms have less than 90-degree view angles from the viewing directions of the mosaics. Note that in the current implementation, only the patches visible in the leftmost (i.e., the reference) mosaics are considered.

\subsection{Plane merging}

After the plane parameters with the smallest SAD value have been obtained for each region, we will have a close look at the best SAD of each region. If the SAD value is less than a preset threshold, then the patch is marked as reliable. We 
have found that a large number of small regions around a large region corresponding to a surface (or part) of a 3D object are generated by color segmentation, and they are difficult to obtain accurate 3D estimates because of the lack of sufficient feature points. Therefore, we perform a modified version of the neighboring plane parameter hypothesis approach proposed by Tao et $\mathrm{al}^{20}$ to infer better plane estimates. The main modification is that the parameters of a neighboring region are adopted only if it is marked reliable and the best neighboring plane parameters are accepted only when the match evaluation cost using the parameters is less than a threshold. The neighboring regions sharing the same plane parameter are then merged into one reliable region. This step is performed recursively till no more merges occur. In this step we prefer to have false negatives than false positives, and the former will be handled in the next stage moving object detection, which is our second major goal. We assume that a moving object is visible in most of the mosaics, so it could be readily detected during a 2D range search illustrated in the next section.

\subsection{Moving object detection}

After the plane merging stage, most of the small regions are merged together and marked as reliable. Moving object patches that move along epipolar lines should obtain reliable matches after the plane merging step, but they appear to be "floating" in air on below the surrounding ground, with depth discontinuities all around it. In other words, they can be identified by checking their 3D anomalies.

The remaining regions with unreliable marks fall into the following two categories: (1) moving objects with motion not obeying the pushbroom epipolar geometry; (2) occluded or partially occluded regions (usually those with dramatically different views across the multi-view mosaics), or regions with large illumination changes. For regions in the second category, their SADs in stereo matching evaluation are always very high. The regions in the first category correspond to those moving objects that do not move in the direction of camera motion, therefore they do not obey the pushbroom stereo epipolar geometry. Therefore, for each of these regions, we perform a 2D-range search within its neighborhood area, and a global match step similar to the first step of normal stereo matching is carried out for each such patch. If a good match (i.e., with a small SAD) is found within the 2D search range, then the region is marked as a moving object.

\section{CB3M: CONTENT-BASED 3D MOSAICS}

The proposed content-based 3D mosaic (CB3M) representations ${ }^{22}$ are highly compressed visual representations for very long video sequences of dynamic 3D scenes. The representations could fit into the MPEG-4 standard ${ }^{13}$, in which a scene is described as a composition of several Video Objects (VOs), encoded separately.

A CB3M representation is a set of $\mathrm{VO}$ primitives (patches) that are defined as

$$
\mathbf{C B 3 M}=\left\{\mathrm{VO}_{\mathrm{i}}, \mathrm{i}=1, \ldots, \mathrm{N}\right\}=\left\{\left(\mathrm{c}_{\mathrm{i}}, \mathbf{b}_{\mathrm{i}}, \mathbf{n}_{\mathrm{i}}, \mathbf{m}_{\mathrm{i}}\right), \mathrm{i}=1, \ldots, \mathrm{N}\right\}
$$

where

(1) $\mathrm{N}$ is the number of VOs, i.e., natural patches (regions);

(2) $\mathrm{c}_{\mathrm{i}}$ is the color ( 3 bytes) of the ith region;

(3) $\mathbf{b}_{\mathbf{i}}$ is the $2 \mathrm{D}$ boundary of the ith region in the left mosaic, chain-coded as $\mathbf{b}_{\mathbf{i}}=\left\{\left(\mathrm{x}_{0}, \mathrm{y}_{0}\right), \mathrm{K}_{\mathrm{i}}, \mathrm{b} 1, \mathrm{~b} 2, \ldots \mathrm{b}_{\mathrm{Ki}}\right\}$, where the starting point $\left(\mathrm{x}_{0}, \mathrm{y}_{0}\right)$ has 4 bytes, and each chain code has 3 bits. $\mathrm{K}_{\mathrm{i}}$ is the number of boundary points (which needs 4 bytes for each boundary) and $\mathrm{K}=\sum \mathrm{K}_{\mathrm{i}}$ is the total for all regions;

(4) $\mathbf{n}_{\mathbf{i}}=\left(\mathrm{n}_{\mathrm{x}}, \mathrm{n}_{\mathrm{y}}, \mathrm{n}_{\mathrm{z}}, \mathrm{d}\right)$ represents the plane parameters of the region in 3D, 4 bytes for each parameter; and

(5) $\mathbf{m}_{\mathbf{i}}$ represents the $\mathrm{L}$ motion parameters of the region if in motion (e.g. $\mathrm{L}=2$ for $2 \mathrm{D}$ translation on the ground).

Therefore the total data amount is

$$
\mathrm{N}_{\text {color }}+\mathrm{N}_{\text {boundaryr }}+\mathrm{N}_{\text {structure }}+\mathrm{N}_{\text {motion }}=3 \mathrm{~N}+(8 \mathrm{~N}+3 \mathrm{~K} / 8)+4 * 4 \mathrm{~N}+4 \mathrm{~L} * \mathrm{~N}_{\mathrm{m}}=27 \mathrm{~N}+3 \mathrm{~K} / 8+4 \mathrm{LN}_{\mathrm{m}} \text { (bytes) }
$$

when each of the motion and structure parameters needs 4 bytes. In the above equation, $\mathrm{N}_{\mathrm{m}}$ is the number of moving regions (which is much smaller than the total region number $\mathrm{N}$ ). 


\section{EXPERIMENTAL RESULTS}

Since it's difficult to obtain ground truth data for real video sequences, a simulated video sequence was generated with the ground truth data for the purpose of algorithm evaluation. Then, the proposed approach for the content-based 3D mosaic representations was applied to multiple stereo mosaics generated from a real world video sequence.

\subsection{Results and analysis on a simulated scene}

Nine parallel-perspective stereo mosaics were generated from a simulated video sequence of a simulated scene with ground truth data of both 3D and moving targets (Fig. 4). The sequence was generated using the following parameters. The virtual "aircraft" with a video camera flied at a 300-meter height above the scene along a 1D translational direction, and the motion direction is perpendicular to the optical axis of the camera. The focal length of the camera is 3000 pixels (as in Eqs. 1 and 3), and the camera moves with a constant speed. The 3D "buildings" are with heights from 5 to 120 meters above the ground, with different roof shapes (rectangular, round, frontal, ridged, slanting, and/or with small attachments). There are occlusions between buildings. Each of the eight moving objects has a height from 2 to 5 meters, and undertakes a 2D translational motion with constant velocity during the period of the capture of the total 1640 frames of images, except the one labeled as "1" in Fig 4a. The velocity of the motion of each moving target is represented in centimeter $(\mathrm{cm})$ per frame. Nine 1-column width slit windows are used to generate the nine mosaics (refer to Fig. 3), every pair of the two consecutive windows has a 40-pixel distance, and hence the total distance between the first and the last windows is 320 pixels. Fig. 4 shows three of the nine mosaics, (a) the leftmost, (b) the center, and (c) the rightmost views. Varying occlusions/visibilities can be seen in these mosaics. The change of velocity of the $1^{\text {st }}$ moving target can be seen from the varying sizes of its images in the three mosaics.
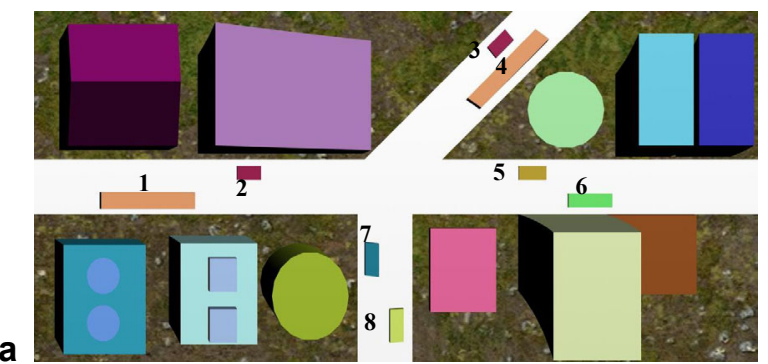

a

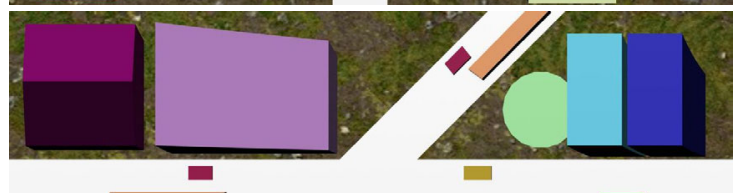

$\square$

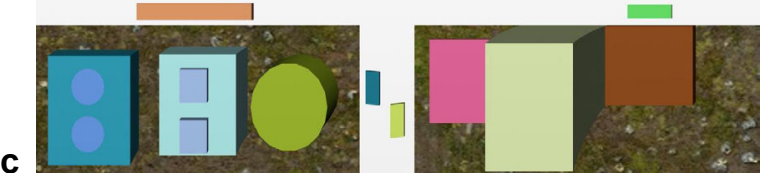

C

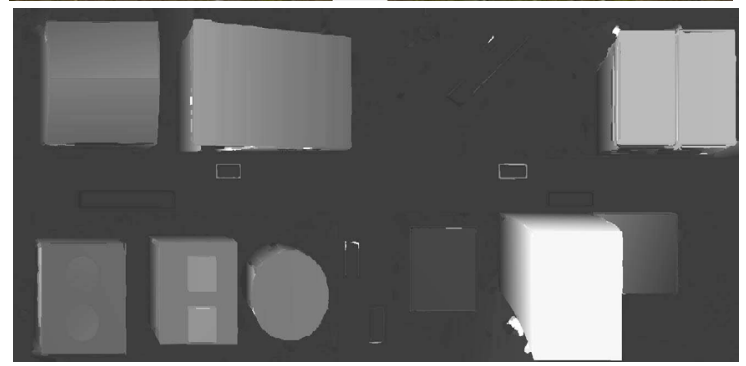

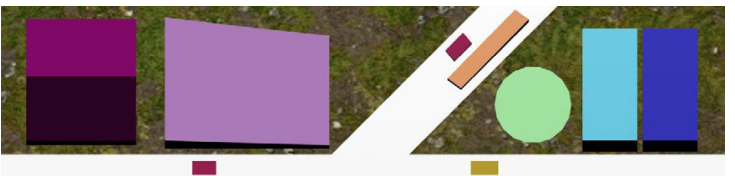

$\square$

-

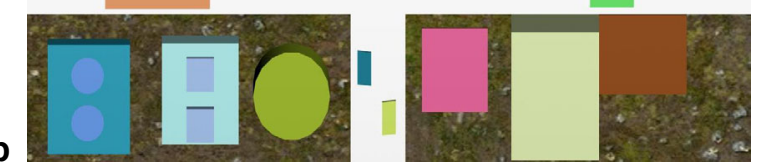

b
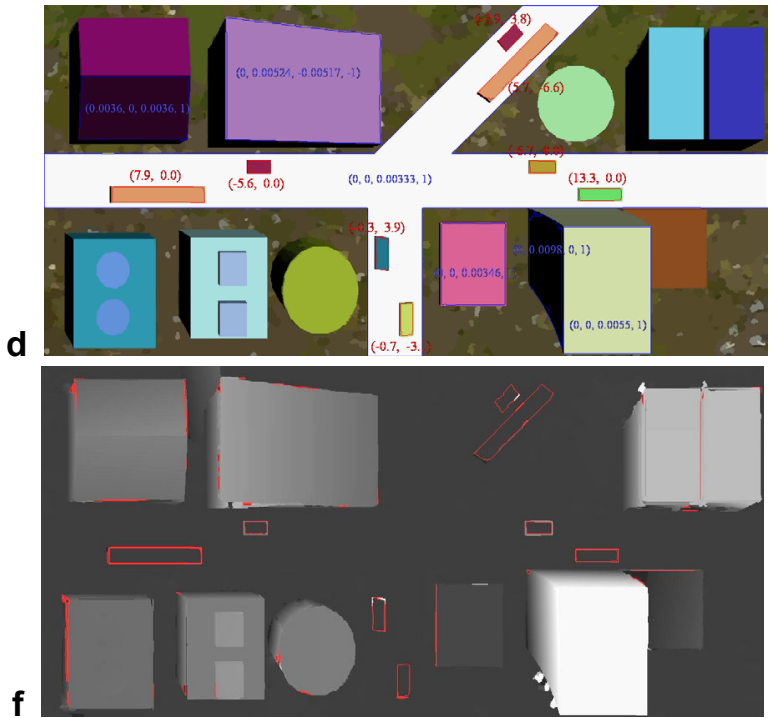

Fig 4. (a) The leftmost, (b) center and (c) rightmost views of the nine mosaics of a simulated scene. The final CB3M representation is shown in (d). Each region is rendered by its average color. Plane parameters (a,b,c,d) (in blue) and boundaries for several representative surfaces, and motion displacements $\left(s_{x}, s_{y}\right)$ (in red) of the detected moving targets are labeled in (d). For comparison, (e) and (f) show the rendered "height" maps of the scene from the stereo matching results from the $1^{\text {st }}$ stereo pair only, and from all the mosaics, respectively. Finer and more accurate results are obtained in (f). 
From the nine mosaics, we use the leftmost mosaic as the reference image to match with the other eight mosaics. For each region in the reference mosaic, there are 8-plane estimation results, and the best estimate is selected for the 3D parametric representation of the region. The final "height" map (Fig. 4f) is rendered as a map of heights of objects from the ground, i.e. $-H \Delta y / d_{y}$, (normalized to a range from 0 to 255 for display). For comparison, we have also generated a height map (Fig. 4e) from the stereo matching results of only the first and the second mosaics (without region merging). It can be seen that by using the best parameter selections from multi-view mosaics and utilizing the plane merging step, finer 3D results are obtained for many building roofs, and more accurate results are obtained for sides of buildings.

We also compare the final estimated height map with the ground truth data. The error histogram is shown in Fig. 5a for all the regions (including the moving object regions and other obvious wrong matches). From the error distribution, we have found that the errors of $86.5 \%$ points in the reference mosaic are within \pm 4 meters. The absolute average value of the errors for those points is only 0.317 meters. Note that in theory, the error of the depth/height estimation by the pushbroom stereo in Eq. 2 can be calculated as $\delta Z=\left(H / d_{y}\right) \delta y$, where $\delta y$ is the error in stereo matching (in pixels). In this experiment, $H$ is 300 meters, and $d_{y}$ is from 40 to 320 pixels (from the first pair to the $8^{\text {th }}$ pair of stereo mosaics), and ideally $\delta y$ is 0.1 pixels with the sub-pixel local match step. Therefore, the theoretical errors after local match go from 0.75 down to about 0.1 meters from the first pair to the $8^{\text {th }}$ pair. However, larger viewing differences introduce larger errors in $\delta y$, therefore the error reduction by using larger disparities (from 40 to 320) is not as significant as the theoretical estimation. On the other hand, plane fitting on the multiple interest points with sub-pixel accuracy increases the accuracy in $\delta Z$, which leads to a more realistic error range close to the average error of the estimated depths/heights in this experiment (i.e., $0.317 \mathrm{~m}$ ).

After the regions have been merged, we analyze all the reliable regions, and those with obvious 3D anomalies are marked as moving objects (along the epipolar lines). For example, in Fig. 4a, the heights of the regions labeled 1 and 6 , if treated as static objects, are estimated as -39 meters and -50 meters high from the ground, respectively, much lower than the ground plane. The regions labeled 2 and 5 are estimated as 94 meters and 98 meters high from the ground, respectively, much higher than the ground. In fact all these regions only are 2 to 5 meters high from the ground. So these regions with such 3-D "anomalies" if incorrectly treated as static objects are detected as moving targets.

On the other hand, those unreliable regions (as possible candidates for moving objects not along the epipolar lines) further go through 2D-range searches for matches within their neighborhood areas (e.g., 30x30 2D range). In Fig. 4a, regions 3, 4, 7 and 8 are moving targets. They do not obtain reliable matches in the stereo match step, but could find reliable matches from their $2 \mathrm{D}$ range searches, between the first mosaic and the rest mosaics. Therefore they are considered as moving targets. Note that those regions marked with red boundaries in the height map have good matches in their 2-D range searches; however, many of them have very small sizes, or have very thin structures, therefore are not considered to be moving targets. The estimated motion parameters $\left(s_{x}, s_{y}\right)$ (in pixels) of those detected moving targets from the first pair of stereo mosaics are marked on the CB3M map in Fig. 4d. The error analysis results of the 8 detected moving targets are shown in Fig. 5b. The average error of the $2 \mathrm{D}$ motion estimation is $(0.198,0.008)$ in velocity $(\mathrm{cm} /$ frame $)$, or $(0.791,0.033)$ in displacements (pixels) between the first pair of the stereo mosaics. The error for the $1^{\text {st }}$ object is the largest since its velocity is not constant.

a

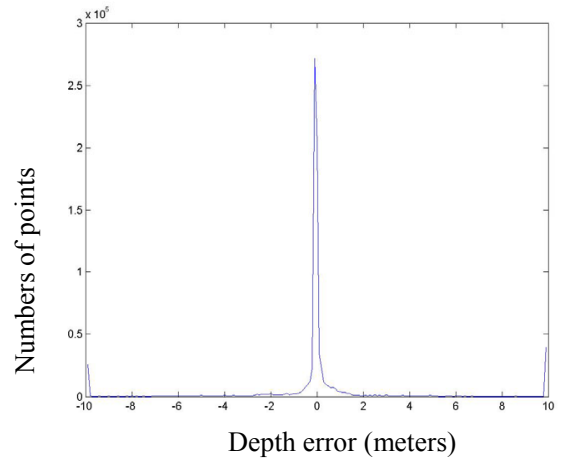

b

\begin{tabular}{|c|c|c|c|c|c|c|}
\hline $\begin{array}{l}\text { Obj } \\
\text { Idx }\end{array}$ & \multicolumn{2}{|c|}{$\begin{array}{l}\text { Ground Truth } \\
(\mathrm{cm} / \text { frame })\end{array}$} & \multicolumn{2}{|c|}{$\begin{array}{l}\text { Estimated Results } \\
(\mathrm{cm} / \text { frame })\end{array}$} & \multicolumn{2}{|c|}{$\begin{array}{c}\text { Errors } \\
\text { (cm/frame) }\end{array}$} \\
\hline & Sx & Sy & Sx* & Sy* & $\Delta \mathrm{Sx}$ & $\Delta \mathrm{Sy}$ \\
\hline 1 & 0 & 2.485 & 0 & 1.649 & 0 & 0.836 \\
\hline 2 & 0 & -1.4 & 0 & -1.628 & 0 & 0.129 \\
\hline 3 & 1.064 & -1.262 & 1.053 & -1.08 & 0.011 & -0.181 \\
\hline 4 & -1.414 & & -1.444 & 1.247 & 0.031 & 0.166 \\
\hline 5 & 0 & -1.999 & 0 & -2.012 & 0 & 0.013 \\
\hline 6 & 0 & 2.499 & 0 & 2.495 & 0 & 0.003 \\
\hline 7 & 0.999 & 0 & 0.982 & -0.076 & 0.017 & 0.076 \\
\hline 8 & -0.781 & 0 & -0.789 & -0.178 & 0.007 & 0.178 \\
\hline
\end{tabular}

Fig. 5. Error analysis. (a) Depth error histogram; (b) motion estimation accuracy 
The compression of a video sequence comes from two steps: stereo mosaicing and then content extraction. For the simulated image sequence, we have 1640 frames of $640 * 480$ color images, so the data amount is $1441 \mathrm{MB}$. The size of pair of the stereo mosaics is $1320 * 640 * 2$, which has $4.83 \mathrm{MB}$ (without compression). The two mosaics in high-quality JPEG format only have $2 * 75 \mathrm{~KB}$; therefore, a compression ratio of about 9,837 is achieved for the stereo mosaics (the first step). If all the nine mosaics are saved for mosaic-based rendering ${ }^{21}$, the data amount will be $9 * 75 \mathrm{~KB}$ hence the compression ratio is about 2,186.

Then after color segmentation, 3D planar fitting and motion estimation, we obtained the CB3M representation (Fig. 4d) of the video sequence, with the total number of the natural regions $\mathrm{N}=1,342$ and the total number of boundary points $\mathrm{K}$ $=119,477$. The total amount of data in its CB3M representation is $80.8 \mathrm{~KB}$ (with a header). This real file size is consistent with the estimation of data amount using Eq. (7), which is about $79.2 \mathrm{~KB}$. The data amount is reduced to 19.4 $\mathrm{KB}$ with a simple lossless Winzip on the $\mathrm{CB} 3 \mathrm{M}$ data; therefore, the compression ratio is about $\mathbf{7 6 , 0 6 1}$. Note that the compression ratio depends on how fine is the color segmentation. In the example shown in Fig. 4d, the main visual features of the scene are coded. More importantly, the CB3M representation has object contents which can be used for object indexing, retrieval and image-based rendering. The plane parameters $(a, b, c, d)$ for the several representative regions are shown on the CB3M map in Fig. 4d (from left to right: one side of a ridged roof, a slanting roof, ground with depth $\mathrm{Z}=300.0 \mathrm{~m}$, roof of a low building with $\mathrm{Z}=289.0 \mathrm{~m}$, and side and roof of a tall building with $\mathrm{Z}=180.0 \mathrm{~m}$ ).

\subsection{Results on real video data}

We also have performed experiments on a real video sequence when the airplane was about 300 meters above the ground. Nine mosaics were generated from the aerial video captured. Fig. 6a shows a pair of stereo mosaics from the nine mosaics, and a close-up window marked in the stereo mosaics, which includes both various 3D structures and moving objects (vehicles). Fig. 6b is a "height" map of that window generated using the proposed method. Note the sharp depth boundaries are obtained for the buildings with different heights and various roof shapes. The moving objects that have been detected across all the nine mosaics are shown by their boundaries (in red). The CB3M mosaic (of the small portion) is shown in Fig. 6c, with a color, a boundary, plane parameters and a motion vector (if in motion) for each patch (region).

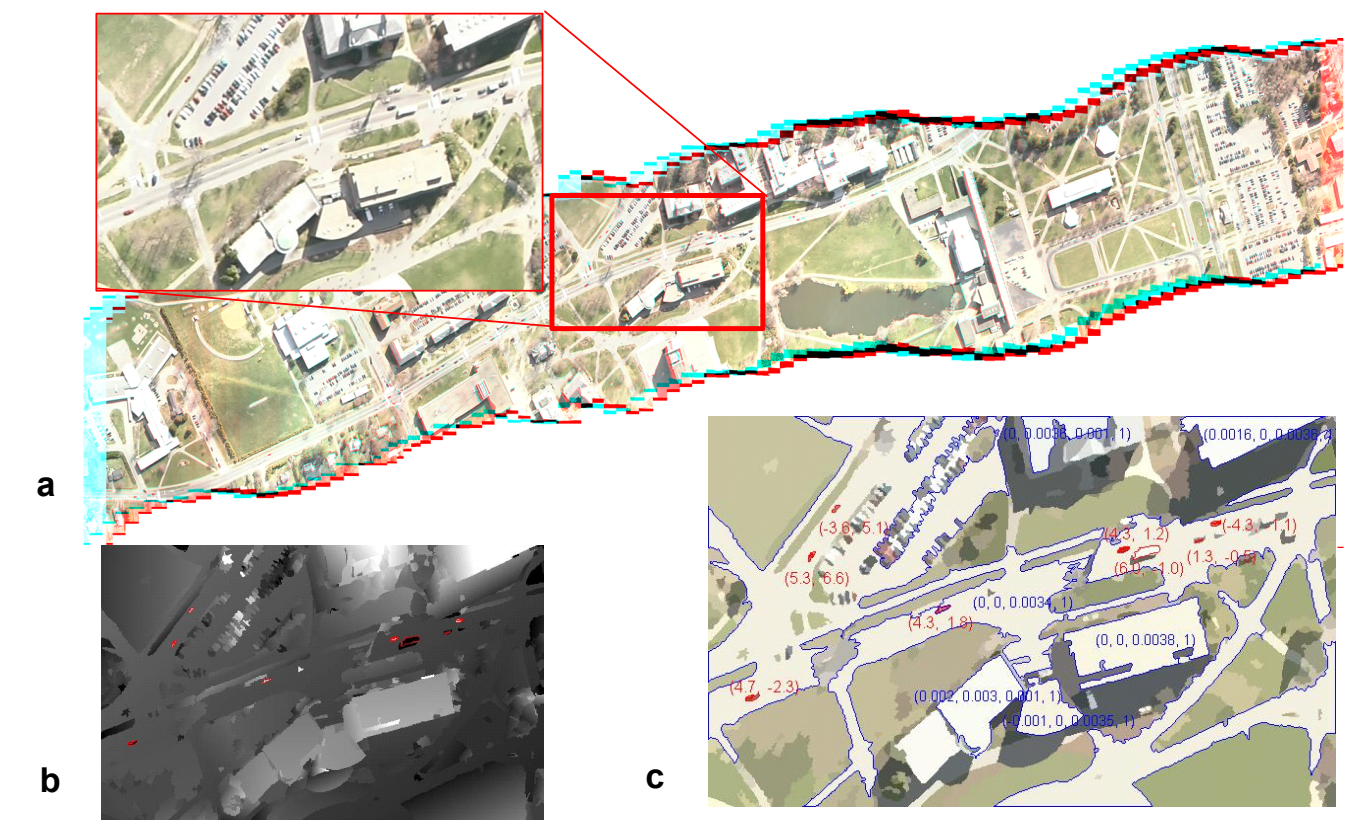

Fig 6. Content-based 3D mosaic representation of an aerial video sequence. (a) A pair of stereo mosaics from the total nine mosaics and a close-up window; (b) the height map of the objects inside that window; (c) the CB3M representation with some of the regions labeled by their boundaries and plane parameters (in blue), and the detected moving targets marked by their boundaries and motion vectors (in red). 
Again we examine the compression of the real video sequence from two steps: stereo mosaicing and then content extraction. For the real image sequence, we have 1000 frames of $640 * 480$ color images, so the data amount is 879 MB. The size of pair of the stereo mosaics (Fig. 6a) is $4448 * 1616 * 2$, which has $41 \mathrm{MB}$ (without compression and with more than half empty space due to the fact that the mosaics go in a diagonal direction). The two mosaics in high-quality JPEG format only have $2 * 560 \mathrm{~KB}$; therefore, a compression ratio of about 800 is achieved for the stereo mosaics (the first step). If nine mosaics are all saved for mosaic-based rendering, then the data amount is $9 * 560 \mathrm{~KB}$ so the compression ratio will be 179 .

Then after color segmentation, 3D planar fitting and motion estimation, we obtained the CB3M representation of the video sequence, with the total number of the natural regions $\mathrm{N}=6,112$ and the total number of boundary points $\mathrm{K}=$ 420,445. The total amount of data in its CB3M representation is $316 \mathrm{~KB}$ (with a header). This real file size is consistent with the estimation of data amount using Eq. (7), which is about $315 \mathrm{~KB}$. The data amount is reduced to $90 \mathrm{~KB}$ with a simple lossless Winzip on the CB3M data; therefore, the compression ratio is about 10,001. Note that the CB3M representation in Fig. 6c consists of regions corresponding to rather large object surfaces in order to rapidly obtain robust 3D structures. However, fine details are not preserved. In our previous work ${ }^{22}$, we over-segmented the reference mosaic so that finer details of the scene can be coded. In that case the compression ratio was still over 2000.

\section{CONCLUSIONS}

In this paper we propose to construct a content-based 3D mosaic representation (CB3M) for long video sequences of 3D and dynamic scenes captured by a camera on a mobile platform. In real applications, the motion of the camera should have a dominant direction of motion (as on an airplane or ground vehicle), but 6 DOF motion is allowed. In the first step, multiple parallel-perspective (pushbroom) mosaics are generated to capture both the 3D and dynamic aspects of the scene under the camera coverage. In the second step, a multi-view, segmentation-based stereo matching algorithm is applied to extract parametric representation of the color, structure and motion of the dynamic and/or 3D objects, and to represent them as planar surface patches.

The content-based 3D mosaic $(\mathrm{CB} 3 \mathrm{M})$ representation is a highly compressed visual representation for very long video sequences of dynamic 3D scenes. It could fit into the MPEG-4 standard, in which a scene is described as a composition of several Video Objects (VOs), encoded separately. The compression of a video sequence comes from both steps: stereo mosaicing and then content extraction. For both simulated and real image sequences of large-scale cultural scenes with many man-made buildings and vegetations, with more than 1000 frames of $640 * 480$ color images, a compression ratio of thousands to ten thousands is achieved. More importantly, the CB3M representation has object contents represented.

In the $\mathrm{CB} 3 \mathrm{M}$ representation presented in this paper, however, many details and practical issues have not been considered. First, more experiments are needed with both simulated and real video sequences to evaluate the coding and compression capabilities of this representation. Second, in order to use the CB3M representations for real applications, further enhancements are also needed. For example, in the current implementation, only 3D parametric information of planar patches in a single reference mosaic is obtained. Since different visibilities are shown in mosaics with different viewing directions, we want to extend the approach presented in the paper to produce multiple depth maps with multiple reference mosaics and then integrate the results by performing occlusion analysis. Finally, developing higher-level representations that group the lower-level natural patches for physical objects may also be very useful for many applications. For example, the neighboring regions, which have been extracted in the patch and interest point extraction stage, and which are important in object recognition and occlusion handling in image rendering, are not represented in the current model.

\section{ACKNOWLEDGEMENTS}

This work is supported by the Air Force Research Lab under the AFRL/SN RASER program for Multi-Sensor Registration (Award No. FA8650-05-1-1853) and an AFRL/HECB Grant for Multimedia Surveillance (Award No. F33615-03-1-63-83). The work is also supported by Army Research Office through Grant No. W911NF-05-1-0011 and by New York Institute for Advanced Studies. Some data were captured in the UMass Computer Vision Lab under an NSF grant (No EIA-9726401). The U.S. Government is authorized to reproduce and distribute reprints for Governmental 
purposes notwithstanding any copyright notation thereon. However, the views and conclusions contained herein are those of the authors and should not be interpreted as necessarily representing the official policies or endorsements, either expressed or implied, of the U.S. Government.

\section{REFERENCES}

1. M. Irani, P. Anandan, J. Bergen, R. Kumar, and S. Hsu, Mosaic representations of video sequences and their applications. Signal Processing: Image Communication, vol. 8, no. 4, May 1996.

2. S. Hsu, P. Anandan, Hierarchical representations for mosaic based video compression, In Proc. Picture Coding Symp., 395-400, March 1996.

3. F. Odone, A. Fusiello and E. Trucco, Robust motion segmentation for content-based video coding, the 6th Conference on Content-based Multimedia Information Access, College de France, 2000: 594-601.

4. W. H. Leung and T. Chen, Compression with mosaic prediction for image-based rendering applications, IEEE Intl. Conf. Multimedia \& Expo., New York, July 2000.

5. Y. Li, H.-Y. Shum, C.-K. Tang, R. Szeliski, Stereo reconstruction from multiperspective panoramas. IEEE Trans. on PAMI, 26(1), 2004: pp 45-62.

6. C. Sun and S. Peleg, Fast Panoramic Stereo Matching using Cylindrical Maximum Surfaces, IEEE Trans. SMC Part B, 34, Feb. 2004: 760-765.

7. J. Xiao and M. Shah, Motion layer extraction in the presence of occlusion using graph cut, In Proc. CVPR'04.

8. Y. Zhou and H. Tao, A background layer model for object tracking through occlusion," In Proc. ICCV'03: 10791085.

9. Q. Ke and T. Kanade, A subspace approach to layer extraction, In Proc. IEEE CVPR'01.

10. Z. Zhu, E.. Riseman, A. Hanson, Generalized Parallel-Perspective Stereo Mosaics from Airborne Videos, IEEE Trans. PAMI, 26(2), Feb 2004, 226-237.

11. Z. Zhu, H. Tang, B. Shen, G. Wolberg, 3D and Moving Target Extraction from Dynamic Pushbroom Stereo Mosaics, IEEE Workshop on Advanced 3D Imaging for Safety and Security (with CVPR'05), June 25, 2005, San Diego, CA, USA.

12. D. Comanicu and P. Meer, Mean shift: a robust approach toward feature space analysis. IEEE Trans. PAMI, May 2002.

13. R. Koenen, F. Pereira and L. Chiariglione, MPEG-4: Context and objectives. Signal Processing: Image Communications, 9(4), 1997:295-300.

14. B. Triggs, P. McLauchlan, R. Hartley and A. Fitzgibbon, Bundle Adjustment -- A Modern Synthesis, In Vision Algorithms: Theory and Practice, Lecture Notes in Computer Science, vol 1883, pp 298--372, 2000, eds. B. Triggs, A. Zisserman and R. Szeliski", Springer-Verlag.

15. R. Gupta and R. Hartley, Linear pushbroom cameras, IEEE Trans PAMI, 19(9), Sep. 1997: 963-975.

16. Z. Zhu, E. M. Riseman, A. R. Hanson and H. Schultz, An Efficient Method for Geo-Referenced Video Mosaicing for Environmental Monitoring. Machine Vision Applications, 16(4), 2005, 203-126

17. M. Okutomi and T. Kanade, 1993. A multiple-baseline stereo," IEEE Trans. PAMI, vol. 15, no. 4, pp. 353-363

18. D. Scharstein and R. Szeliski. A Taxonomy and Evaluation of Dense Two-Frame Stereo Correspondence Algorithms. IJCV 47(1/2/3):7-42, April-June 2002

19. G. Medioni, S. Kang, Emerging Topics in Computer Vision. Prentice Hall, ISBN: 0131013661, 2004

20. H. Tao, H. S. Sawhney and R. Kumar, 2001. A global matching framework for stereo computation, In Proc. ICCV'01

21. Z. Zhu, A. R. Hanson, Mosaic-Based 3D Scene Representation and Rendering, Special Session on Interactive Representation of Still and Dynamic Scenes, the Eleventh International Conference on Image Processing, Genova, Italy, September 11-14, 2005, pp I-633 -636.

22. Z. Zhu, H. Tang, G. Wolberg and J. R. Layne, Content-Based 3D Mosaic Representation for Video of Dynamic 3D Scenes. IEEE/AIPR Workshop 2005: Multi-Modal Imaging, Washington DC, October 19-21, 2005 